\title{
Nowak, Eva (Hrsg.): Accreditation and assessment of journalism education in Europe: quality evaluation and stakeholder influence (Aktuell. Studien zum Journalismus, Band 15)
}

\author{
Baden-Baden: Nomos 2019. 241 Seiten. Preis: $€ 49$
}

\section{Tina Bettels-Schwabbauer}

Online publiziert: 18. Juni 2020

(C) Der/die Autor(en) 2020

Die Eigenschaften „,aufwendig“, „,ürrokratisch“, „umständlich“ kommen vielen JournalistenausbilderInnen als erstes in den Sinn, wenn sie an die Akkreditierung von Ausbildungsangeboten denken. Selbst die Herausgeberin des Bandes Eva Nowak, Professorin für Journalismus an der Jade Hochschule in Wilhelmshaven, räumt in ihrer Einleitung gleich im ersten Satz ein, dass das Thema für sie nicht sehr ansprechend klang, als sie 2013 in einer Arbeitsgruppe auf dem World Journalism Education Congress in Mechelen zum ersten Mal damit konfrontiert wurde.

Bereits im zweiten Satz zeigt sie aber auf, warum sie sich nun doch mit dem Thema beschäftigt und ihm auch gleich einen ganzen Sammelband widmet: Akkreditierung ist ein entscheidender Faktor dafür, wie die Journalistenausbildung in einem Land strukturiert und umgesetzt wird und wie abhängig sie von Akteuren wie dem Staat, der Medienindustrie und anderen einflussreichen Gruppierungen ist.

So zeigt der Sammelband anhand von zwölf Länderstudien nicht nur, wie Journalistenausbildung in Europa akkreditiert und evaluiert wird, sondern auch, wie staatliche Organisationen und die Medienindustrie durch ihre Akkreditierung die Ziele, Inhalte und Strukturen der Ausbildung beeinflussen, Qualitätsstandards setzen und damit gleichzeitig in die Autonomie der Journalistenausbildung eingreifen.

Mit Beiträgen aus Deutschland, Finnland, Frankreich, Georgien, Großbritannien, Irland, den Niederlanden, Rumänien, Russland, der Schweiz, Spanien und Ungarn sind Länder aus ganz Europa vertreten. Sie haben gemeinsam, dass sie alle Mitglieder des Europarats und des Europäischen Hochschulraums (EHEA) sind - in punkto ihrer historischen Entwicklung, politischen Umgebung und Angebote der journalistischen Ausbildung weisen sie aber erhebliche Unterschiede auf.

T. Bettels-Schwabbauer $(\bowtie)$

Institut für Journalistik, TU Dortmund, Emil-Figge-Str. 50, 44227 Dortmund, Deutschland

E-Mail: tina.bettels@tu-dortmund.de 
Insofern sorgt die Auswahl für einen sehr guten Ein- und Überblick, wie sich vorgeschriebene Standards und Verfahren hinsichtlich der Akkreditierung unterscheiden und welche Interessengruppen Einfluss auf den Prozess und damit auf die journalistische Ausbildung nehmen. Neben der Akkreditierung von Studiengängen wird in den Länderbeiträgen auch - wo vorhanden - auf die Akkreditierung und Qualitätsbewertung außeruniversitärer Aus- und Fortbildung eingegangen.

Der Sammelband dürfte auch LeserInnen interessieren, die sich nicht mit allen Akkreditierungsdetails auseinandersetzen möchten, bieten die Beiträge doch auch einen aktuellen Überblick über das journalistische Ausbildungsangebot in dem jeweiligen Land und darüber, welche Wege in den Journalismus führen. Während in Finnland, Georgien, den Niederlanden, Spanien, Rumänien, Russland und inzwischen auch Irland die akademische Journalistenausbildung überwiegt, werden in Frankreich JournalistInnen hauptsächlich an Journalistenschulen (die allerdings oft an Universitäten angesiedelt sind) ausgebildet. In Deutschland, der Schweiz und Großbritannien wird mit der journalistischen Ausbildung an Hochschulen, an Journalistenschulen und in Medienunternehmen die breiteste Palette angeboten.

Ungarn sticht in Sachen hochschulgebundener Journalistenausbildung heraus: Dort gibt es, wie Tibor Mester und Annamária Torbó in ihrem Beitrag erläutern, „Journalismus" nicht als eigenständigen Studiengang, sondern lediglich als Spezialisierung in einigen kommunikations- und medienwissenschaftlichen Studiengängen, die zumeist sehr theorie- und forschungsorientiert sind. Die beiden AutorInnen merken auch an, dass zum Thema Journalistenausbildung in Ungarn noch nicht viel geforscht wurde - umso wichtiger finde ich es, dass in diesem Sammelband ein Beitrag über Ungarn enthalten ist, der nicht nur den Prozess der Akkreditierung von Studiengängen, in denen journalistische Inhalte vermittelt werden, aufzeigt, sondern auch die Probleme der Journalistenausbildung in dem Land darstellt.

Wie in den USA durch den Accrediting Council on Education in Journalism and Mass Communications (ACEJMC) Journalismusstudiengänge akkreditiert werden, war dagegen schon oft Gegenstand der Literatur. Schließlich dient die Organisation, der sowohl Medienpraktiker als auch Ausbilder angehören, mit ihrer 75-jährigen Erfahrung als Modell für Akkreditierungsstellen in anderen Ländern, wie Joe Foote im letzten Kapitel des Sammelbands betont. Für einige LeserInnen mag der Blick in die USA das Bild komplett machen, für andere ist das Kapitel ein Anhängsel, das der Band nicht unbedingt gebraucht hätte.

Ein Muss ist aber das vorletzte Kapitel, in dem die Herausgeberin die wichtigsten Aspekte der Länderbeiträge zusammenführt und miteinander vergleicht. Da alle zwölf Länder in diesem Band Mitglieder des Europäischen Hochschulraums sind, überrascht es nicht, dass die Akkreditierung von Studiengängen in diesen Ländern eine ähnliche Struktur aufweist. Die Hauptunterschiede betreffen vorgeschriebene Standards und Prozesse und damit die Autonomie gegenüber staatlicher Einflussnahme. Nicht immer zielt die Akkreditierung nur auf die Verbesserung der Ausbildung, sondern auch auf die Kontrolle von Bildungseinrichtungen ab.

Nowak unterscheidet in zwei Arten von Akkreditierung: Den entwicklungsorientierten Ansatz (,,development-oriented approach“) schreibt sie Deutschland, Finnland, den Niederlanden und der Schweiz zu, den ergebnisorientierten Ansatz (,,output-oriented approach“) Georgien, Rumänien, Russland und Ungarn. In Ländern, in 
denen ein liberaler entwicklungsorientierter Ansatz bei der Akkreditierung verfolgt wird, gesteht man einer Einrichtung zu, eigene Qualitätsstandards für die Ausbildung zu entwickeln. Ein ergebnisorientierter Ansatz dagegen zielt vor allem auf die Kontrolle des Qualitätsmanagements und des Outputs ab. Dieses regulierende Vorgehen ist typisch für eher autoritär geprägte Länder - und für Länder, in denen Vertreter der Medienindustrie journalistische Ausbildungsangebote akkreditieren, wie in Frankreich und Großbritannien. In Spanien existieren für die Akkreditierung journalistischer Studiengänge detaillierte Kriterien, sie wird aber dezentral organisiert, womit Elemente beider Ansätze enthalten sind.

Die AutorInnen der sehr detailgenauen Länderbeiträge und die Herausgeberin zeigen, dass es sich durchaus lohnt, auch eher trockenen Themen eine Chance zu geben, sind sie doch oft vielschichtiger, als auf den ersten Blick zu erkennen ist.

Funding Open Access funding provided by Projekt DEAL.

Open Access Dieser Artikel wird unter der Creative Commons Namensnennung 4.0 International Lizenz veröffentlicht, welche die Nutzung, Vervielfältigung, Bearbeitung, Verbreitung und Wiedergabe in jeglichem Medium und Format erlaubt, sofern Sie den/die ursprünglichen Autor(en) und die Quelle ordnungsgemäß nennen, einen Link zur Creative Commons Lizenz beifügen und angeben, ob Änderungen vorgenommen wurden.

Die in diesem Artikel enthaltenen Bilder und sonstiges Drittmaterial unterliegen ebenfalls der genannten Creative Commons Lizenz, sofern sich aus der Abbildungslegende nichts anderes ergibt. Sofern das betreffende Material nicht unter der genannten Creative Commons Lizenz steht und die betreffende Handlung nicht nach gesetzlichen Vorschriften erlaubt ist, ist für die oben aufgeführten Weiterverwendungen des Materials die Einwilligung des jeweiligen Rechteinhabers einzuholen.

Weitere Details zur Lizenz entnehmen Sie bitte der Lizenzinformation auf http://creativecommons.org/ licenses/by/4.0/deed.de.

Tina Bettels-Schwabbauer ist wissenschaftliche Mitarbeiterin am Erich-Brost-Institut für internationalen Journalismus und am Institut für Journalistik der Technischen Universität Dortmund. 